Pacific Journal of Mathematics

AN APPLICATION OF DYES THEOREM ON PROJECTIO
LATTICES TO ORTHOGONAL DECOMPOSABLE

HUZIHIRO ARAK 


\title{
AN APPLICATION OF DYE'S THEOREM ON PROJECTION LATTICES TO ORTHOGONALLY DECOMPOSABLE ISOMORPHISMS
}

\author{
HuZIHIRo ARAKI \\ Dedicated to the memory of Professor Henry Abel Dye
}

\begin{abstract}
The predual $M_{*}$ of a von Neumann algebra $M$ is an orthogonally decomposable ordered Banach space in the sense of Yamamuro. By an application of Dye's theorem on projection lattices, it is shown that the isomorphism of this structure is implemented by a Jordan automorphism of $M$ if $M$ is of type $I$ without direct summands of type $I_{2}$ in the large.
\end{abstract}

1. Main results. The notion of orthogonal decomposability (o.d.) of a real Banach space $E$ ordered by a proper closed convex cone $E_{+}$ has been investigated by Yamamuro [14]. The complex Banach space $E+i E$ (with the natural conjugation $(x+i y)^{*}=x-i y$ for $x, y \in E$ ) will also be called o.d. if $E$ is o.d. The predual $M_{*}$ of a von Neumann algebra $M$ is an o.d. (complex) ordered Banach space, in which the relevant orthogonal decomposition of $\omega=\omega^{*} \in M_{*}$ is $\omega=\omega_{+}-\omega_{-}$ with $\omega_{ \pm} \in M_{*}^{+}$and $\left(\operatorname{supp} \omega_{+}\right) \perp\left(\operatorname{supp} \omega_{-}\right.$) (supp denotes the support projection) and is unique (for example, Theorem 3.2.7 in [3]).

An order preserving continuous linear map of an o.d. Banach space $E$ is called an o.d. homomorphism if it preserves the orthogonal decomposition. Such o.d. homomorphisms of an ordered Hilbert space have been investigated in [5], [6]. We shall call an o.d. homomorphism an o.d. isomorphism if it is an order isomorphism. In this note, we make some comments on an o.d. isomorphism of the predual $M_{*}$ of a von Neumann algebra $M$. Our main result is the following theorem.

TheOREM 1.1. Let $M_{i}(i=1,2)$ be $W^{*}$-algebras with one of them type I having no direct summands of type $\mathrm{I}_{2}$ in the large. A map $\alpha$ is an o.d. isomorphism of $\left(M_{1}\right)_{*}$ onto $\left(M_{2}\right)_{*}$, if and only if there exist a positive central element $\lambda_{\alpha}$ of $M_{2}$ with a bounded inverse and a Jordan isomorphism $\beta_{\alpha}$ of $M_{2}$ onto $M_{1}$ such that

$$
\alpha \omega=\omega \beta_{\alpha} \lambda_{\alpha} \quad\left(\forall \omega \in\left(M_{1}\right)_{*}\right) .
$$

We leave the case of a general $M$ as an open problem. 
REMARK 1.2. Any Jordan isomorphism of a $W^{*}$-algebra is a direct sum of a $*$-isomorphism and $*$-antiisomorphism [8]. The central element $\lambda_{\alpha}$ will be a positive number if $M_{1}$ or $M_{2}$ (and hence both) is a factor.

REMARK 1.3. If $\alpha$ is implementable by a linear map $\varphi_{\alpha}$ between the standard representation space of $M_{1}$ and $M_{2}$ in the form

$$
(\alpha \omega)(x)=\omega\left(\varphi_{\alpha}^{*} x \varphi_{\alpha}\right),
$$

then $\alpha$ is of the form (1.1) by a theorem of Dang and Yamamuro ([5], (2.2)). Conversely, any $\alpha$ of the form (1.1) is implemented in the form of (1.2) by such a linear o.d. isomorphism $\varphi_{\alpha}$ where the order is by the natural positive cone of the standard representation space (the multiplication by $\lambda_{\alpha}^{1 / 2}$ and the unitary implementer of $\beta_{\alpha}$ ). Therefore the issue here is the implementability.

Specializing Theorem 1.1 to a norm preserving $\alpha$, we obtain the following generalization of Wigner's theorem $[13,1,2,12,11]$.

COROLlaRy 1.4. Let $M_{i}(i=1,2)$ be $W^{*}$-algebras with one of them type I having no direct summands of type $\mathrm{I}_{2}$ in the large. A bijective map $\alpha$ of $\left(M_{1}\right)_{* 1}^{+}$onto $\left(M_{2}\right)_{* 1}^{+}$is affine and preserves orthogonality of states if and only if there exists a Jordan isomorphism $\beta_{\alpha}$ of $M_{2}$ onto $M_{1}$ such that

$$
\alpha \omega=\omega \beta_{\alpha} \quad\left(\forall \omega \in\left(M_{1}\right)_{* 1}^{+}\right) .
$$

REMARK 1.5. $\left(M_{i}\right)_{* 1}^{+}$is the set of all normal normalized positive functionals on $M_{i}$, i.e. the state space of $M_{i}$. Its affine structure is based on the physical (or probabilistic) notion of a mixture of states. The orthogonality of two states means that the two states are sharply distinguishable in the following sense: if the system is surely in one of the two states, then it is surely not in the other state. Wigner uses the condition of the preservation of the transition probability between two states. The present version uses only the zero transition probability. Wigner's theorem is for $B(H)$ and here it is generalized to a type I von Neumann algebra possibly with a nontrivial center (with type $I_{2}$ excluded).

Kadison [9] has given a similar theorem for a general von Neumann algebra with our assumption of the preservation of orthogonality for $\alpha$ replaced by the weak $*$ continuity assumption.

Theorem 1.1 is related to an isomorphism between the lattices $P\left(M_{1}\right)$ and $P\left(M_{2}\right)$ of orthogonal projections in $M_{1}$ and $M_{2}$ via the following proposition. 
Proposition 1.6. (1) If $\alpha$ is an o.d. isomorphism of $\left(M_{1}\right)_{*}$ onto $\left(M_{2}\right)_{*}$, then there exists a unique isomorphism $\gamma_{\alpha}$ of the orthocomplemented lattice $P\left(M_{1}\right)$ onto $P\left(M_{2}\right)$ such that

$$
\gamma_{\alpha}(\operatorname{supp} \omega)=\operatorname{supp} \alpha \omega \quad\left(\forall \omega \in\left(M_{1}\right)_{*}^{+}\right) .
$$

(2) Assume that $M_{1}$ does not have any type $\mathrm{I}_{2}$ direct summands in the large. If $\gamma$ is an isomorphism of the complete orthocomplemented lattice $P\left(M_{1}\right)$ onto $P\left(M_{2}\right)$, there exists a unique o.d. isomorphism $\alpha_{\gamma}$ of $\left(M_{1}\right)_{*}$ onto $\left(M_{2}\right)_{*}$ such that

$$
\left(\alpha_{\gamma} \omega\right)(\gamma p)=\omega(p) \quad\left(\forall p \in P\left(M_{1}\right), \omega \in\left(M_{1}\right)_{*}\right) .
$$

(3) If $\gamma$ and $\alpha_{\gamma}$ are as in (2), the $\gamma_{\alpha}$ determined for $\alpha=\alpha_{\gamma}$ as in (1) coincides with the given $\gamma$.

(4) If $\alpha$ is of the form (1.1), $\gamma_{\alpha}$ is determined as in (1) and $\alpha_{\gamma}$ is determined for $\gamma=\gamma_{\alpha}$ as in (2), then

$$
\alpha_{\gamma} \omega=\omega \beta_{\alpha} .
$$

Now the following theorem of Dye ([7], p. 83 Corollary) is applicable to $\gamma$.

THEOREM 1.7 (DYE). Any projection orthoisomorphism of a $W^{*}$ algebra $M$ with no direct summands of type $\mathrm{I}_{2}$ in the large onto a $W^{*}$-algebra $N$ is implemented by the direct sum of $a *$-isomorphism and $a *$-antiisomorphism.

Here a projection orthoisomorphism is a one-one mapping between the set of projections which preserves orthogonality, i.e. $P \perp Q$ if and only if their images are orthogonal. It automatically preserves order and commutativity.

\section{Isomorphisms of projection lattices.}

LEMMA 2.1. If $\alpha$ is an o.d. isomorphism of $\left(M_{1}\right)_{*}$ onto $\left(M_{2}\right)_{*}$, then $\alpha^{-1}$ is an o.d. isomorphism of $\left(M_{2}\right)_{*}$ onto $\left(M_{1}\right)_{*}$.

Proof. Let $\alpha\left(\omega_{1}\right) \perp \alpha\left(\omega_{2}\right)$ for $\omega_{1}, \omega_{2} \in\left(M_{1}\right)_{*}^{+}$. We shall prove that $\omega_{1} \perp \omega_{2}$. (The orthogonality is that of the support projections.)

Let $\omega_{1}-\omega_{2}=\omega_{+}-\omega_{-}$be the Jordan decomposition of the selfadjoint normal linear functional $\omega_{1}-\omega_{2}$ into positive linear functionals with mutually orthogonal support:

$$
\omega_{+} \perp \omega_{-} .
$$


Then, by the o.d. property of $\alpha$, we have

$$
\alpha\left(\omega_{1}\right)-\alpha\left(\omega_{2}\right)=\alpha\left(\omega_{+}\right)-\alpha\left(\omega_{-}\right), \quad \alpha\left(\omega_{+}\right) \perp \alpha\left(\omega_{-}\right) .
$$

By assumption, $\alpha\left(\omega_{1}\right) \perp \alpha\left(\omega_{2}\right)$. By the uniqueness of the orthogonal decomposition, we obtain

$$
\alpha\left(\omega_{1}\right)=\alpha\left(\omega_{+}\right), \quad \alpha\left(\omega_{2}\right)=\alpha\left(\omega_{-}\right) .
$$

Since $\alpha$ is bijective, we obtain $\omega_{1}=\omega_{+}$and $\omega_{2}=\omega_{-}$. In particular, $\omega_{1} \perp \omega_{2}$.

LEMMA 2.2. For any projection $p \in M$,

$$
\begin{aligned}
p & =1-\bigvee_{\omega}\{\operatorname{supp} \omega: \operatorname{supp} \omega \perp p\} \\
& =\bigvee_{\omega}\{\operatorname{supp} \omega: \operatorname{supp} \omega \leq p\} .
\end{aligned}
$$

Proof. Let $H$ be a standard representation space of $M$ and let $p$ be a projection in $M$. Then

$$
1-p=\bigvee_{\xi}\left\{\operatorname{supp} \omega_{\xi}: \xi \in(1-p) H\right\}
$$

where $\omega_{\xi}(x)=(x \xi, \xi)$. This proves $(2.4 \mathrm{a})$. Writing $1-p$ as $p$ in $(2.4 \mathrm{a})$ and using the equivalence of $\operatorname{supp} \omega \perp(1-p)$ and $\operatorname{supp} \omega \leq p$, we obtain (2.4b).

LEMMA 2.3. Let $\alpha$ be an o.d. isomorphism of $\left(M_{1}\right)_{*}$ onto $\left(M_{2}\right)_{*}$. For $\omega_{1}, \omega_{2} \in\left(M_{1}\right)_{*}^{+}$, the following are equivalent.

$$
\begin{aligned}
\operatorname{supp} \omega_{1} & =\operatorname{supp} \omega_{2}, \\
\operatorname{supp} \alpha\left(\omega_{1}\right) & =\operatorname{supp} \alpha\left(\omega_{2}\right) .
\end{aligned}
$$

Proof. By the definition of an o.d. isomorphism and Lemma 2.1, $\omega^{\prime} \perp \omega$ and $\alpha\left(\omega^{\prime}\right) \perp \alpha(\omega)$ are equivalent. Hence

$$
\left\{\omega^{\prime \prime}: \omega^{\prime \prime} \perp \alpha(\omega)\right\}=\left\{\alpha\left(\omega^{\prime}\right): \omega^{\prime} \perp \omega\right\}
$$

for any $\omega \in\left(M_{1}\right)_{*}^{+}$. Since the right-hand side is determined by supp $\omega$, it coincides for $\omega=\omega_{1}$ and $\omega=\omega_{2}$ if (2.6a) holds. Then the equality of the left-hand side together with (2.4a) implies (2.6b). Since $\alpha^{-1}$ is an o.d. isomorphism by Lemma 2.1 , the converse also holds. 
LEMMA 2.4. For any projection $p \in M_{1}$, the following holds:

$$
\begin{aligned}
1 & -\bigvee_{\omega}\{\operatorname{supp} \alpha(\omega): \operatorname{supp} \omega \perp p\} \\
& =\bigvee_{\omega^{\prime}}\left\{\operatorname{supp} \alpha\left(\omega^{\prime}\right): \operatorname{supp} \omega^{\prime} \leq p\right\} \quad\left(\equiv \gamma_{\alpha}(p)\right) .
\end{aligned}
$$

Proof. If $\operatorname{supp} \omega \perp p$ and $\operatorname{supp} \omega^{\prime} \leq p$, then $\operatorname{supp} \omega \perp \operatorname{supp} \omega^{\prime}$ and hence $\operatorname{supp} \alpha(\omega) \perp \operatorname{supp} \alpha\left(\omega^{\prime}\right)$. Hence the inclusion $(2.8 \mathrm{a}) \supseteq(2.8 \mathrm{~b})$ holds. Let $(2.8 \mathrm{a})$ be $p^{\prime}$. For any $\omega^{\prime \prime} \in\left(M_{2}\right)_{*}^{+}$satisfying supp $\omega^{\prime \prime} \leq p^{\prime}$, let $\omega^{\prime}=\alpha^{-1} \omega^{\prime \prime}$. Then $\operatorname{supp} \alpha\left(\omega^{\prime}\right) \perp \operatorname{supp} \alpha(\omega)$ whenever supp $\omega \perp p$. This implies supp $\omega^{\prime} \perp \operatorname{supp} \omega$ by Lemma 2.1 and hence supp $\omega^{\prime} \leq p$ by $(2.4 a)$. Therefore supp $\alpha\left(\omega^{\prime}\right)=\operatorname{supp} \omega^{\prime \prime}$ is contained in $(2.8 \mathrm{~b})$ and hence the inclusion $(2.8 \mathrm{a}) \subseteq(2.8 \mathrm{~b})$ holds by $(2.4 \mathrm{~b})$.

Proof of Proposition 1.6(1). We define $\gamma_{\alpha}$ by (2.8). If $p=\operatorname{supp} \omega^{\prime}$, then $\gamma_{\alpha}(p)=\operatorname{supp} \alpha\left(\omega^{\prime}\right)$ by Lemma 2.1 and (2.4a). Hence (1.4) holds.

If $p_{1} \leq p_{2}$, then (2.8) implies

$$
\gamma_{\alpha}\left(p_{1}\right) \leq \gamma_{\alpha}\left(p_{2}\right) \text {. }
$$

By using $1-p$ for $p$ in (2.8b) and comparing it with (2.8a) for $p$, we obtain

$$
\gamma_{\alpha}(1-p)=1-\gamma_{\alpha}(p) \text {. }
$$

By (2.8b), supp $\omega^{\prime} \leq p$ implies supp $\alpha\left(\omega^{\prime}\right) \leq \gamma_{\alpha}(p)$. Conversely, if $\operatorname{supp} \alpha\left(\omega^{\prime}\right) \leq \gamma_{\alpha}(p)$, then $\alpha\left(\omega^{\prime}\right) \perp \alpha(\omega)$ whenever supp $\omega \perp p$. This implies $\omega^{\prime} \perp \omega$ by Lemma 2.1 and hence $\operatorname{supp} \omega^{\prime} \leq p$ by $(2.4 \mathrm{a})$. Therefore the following are equivalent.

$$
\begin{gathered}
\operatorname{supp} \alpha\left(\omega^{\prime}\right) \leq \gamma_{\alpha}(p), \\
\operatorname{supp} \omega^{\prime} \leq p .
\end{gathered}
$$

If we denote $\alpha^{\prime}=\alpha^{-1}$, then this equivalence and (2.4) imply

$$
\gamma_{\alpha^{\prime}} \gamma_{\alpha}(p)=p, \quad \gamma_{\alpha} \gamma_{\alpha^{\prime}}\left(p^{\prime}\right)=p^{\prime}
$$

(2.9) and (2.12) imply that $\gamma_{\alpha}$ is a lattice isomorphism. Together with (2.10), we see that $\gamma_{\alpha}$ is an isomorphism of orthocomplemented lattices.

The mapping of supp $\omega$ specified by (1.4) together with the structure of the orthocomplemented lattice completely determines the mapping $\gamma_{\alpha}(p)$ by (2.4). Hence the uniqueness of $\gamma_{\alpha}$ follows.

We note that if $M$ is $\sigma$-finite (e.g. $M_{*}$ is separable), then any $p$ is of the form $p=\operatorname{supp} \omega$ for some $\omega \in M_{*}^{+}$and the proof is much shorter. 
3. Proof of Theorem 1.1. The right-hand side of (1.1) is linear and continuous in the norm topology. Since the multiplication of a central element $\lambda_{\alpha}$ does not change the support of states, we have

$$
\operatorname{supp}\left(\omega \beta_{\alpha} \lambda_{\alpha}\right)=\beta_{\alpha}^{-1}(\operatorname{supp} \omega) \text {. }
$$

Since a Jordan isomorphism $\beta_{\alpha}^{-1}$ preserves the orthogonality of projections, the "if" part of Theorem 1.1 follows.

To prove the converse, let $\alpha$ be an o.d. isomorphism of $\left(M_{1}\right)_{*}$ onto $\left(M_{2}\right)_{*}$ and $\gamma_{\alpha}$ be the isomorphism of $P\left(M_{1}\right)$ onto $P\left(M_{2}\right)$ given by Proposition 1.6 (1). Because of the o.d. property of $\alpha$ and Lemma 2.1, $\gamma_{\alpha}$ is an orthoisomorphism and hence can be extended to a Jordan isomorphism of $M_{1}$ onto $M_{2}$ by Dye's Theorem (Theorem 1.7). Let $\beta_{\alpha}$ be the inverse of this Jordan isomorphism and let

$$
\alpha^{\prime} \omega=(\alpha \omega) \beta_{\alpha}^{-1} \text {. }
$$

Then $\alpha^{\prime}$ is an o.d. automorphism of $\left(M_{1}\right)_{*}$ and

$$
\operatorname{supp}\left(\alpha^{\prime} \omega\right)=\beta_{\alpha}(\operatorname{supp} \alpha \omega)=\operatorname{supp} \omega
$$

namely $\alpha^{\prime}$ preserves the support of any $\omega \in\left(M_{1}\right)_{*}^{+}$. Thus we can restrict our attention to the case of $M=M_{1}=M_{2}$ and an o.d. automorphism $\alpha$ of $M_{*}$ which preserves the support of any $\omega \in M_{*}^{+}$.

We now limit ourselves to the case of a type I von Neumann algebra $M$.

LEMMA 3.1. If $\alpha$ is an o.d. automorphism of $M_{*}$ preserving the support of all $\omega \in M_{*}^{+}$and if $\omega$ is such that supp $\omega$ is an abelian projection of $M$, then there exists a central element $\lambda_{\omega}$ with a bounded inverse such that

$$
\alpha \omega=\omega \lambda_{\omega}
$$

Proof. Let $e=\operatorname{supp} \omega$ and $\bar{e}$ be its central support. Then $e M e=e Z$ because $e$ is assumed to be an abelian projection. Here $Z$ is the center of $M$ and $e Z$ is isomorphic to $\bar{e} Z$. Let $\bar{\omega}$ and $\overline{\alpha \omega}$ be the restriction of $\omega$ and $\alpha \omega$ to $Z$. They have a common support $\bar{e}$. Let

$$
\lambda_{\omega}=(1-\bar{e})+d(\overline{\alpha \omega}) / d \bar{\omega}
$$

where the last term is the Radon-Nikodym derivative with support $\bar{e}$.

Let $e_{n}$ be the spectral projection of $\lambda_{\omega}$ for the interval $[n, n+1)$. Since $\alpha$ preserves the support of any $\omega \in M_{*}^{+}$, we obtain

$$
\begin{aligned}
\operatorname{supp} \alpha\left(\omega e_{n}\right) & =\operatorname{supp}\left(\omega e_{n}\right)=e_{n} e, \\
\operatorname{supp} \alpha\left(\omega \cdot\left(1-e_{n}\right)\right) & =\operatorname{supp}\left(\omega \cdot\left(1-e_{n}\right)\right)=\left(1-e_{n}\right) e .
\end{aligned}
$$


Applying these support properties to

$$
\alpha \omega=\alpha\left(\omega e_{n}+\omega \cdot\left(1-e_{n}\right)\right)=\alpha\left(\omega e_{n}\right)+\alpha\left(\omega \cdot\left(1-e_{n}\right)\right),
$$

we obtain

$$
\alpha\left(\omega e_{n}\right)=(\alpha \omega) e_{n}
$$

and hence

$$
\left\|\alpha\left(\omega e_{n}\right)\right\|=(\alpha \omega)\left(e_{n}\right)=\omega\left(\lambda_{\omega} e_{n}\right) \geq n \omega\left(e_{n}\right)=n\left\|\omega e_{n}\right\| .
$$

Therefore $\omega\left(e_{n}\right)=\left\|\omega e_{n}\right\|$ must vanish for $n>\|\alpha\|$. Since $e_{n} \leq \bar{e}=$ $\operatorname{supp} \bar{\omega}$ for $n \neq 1$, we have $e_{n}=0$ for $n>\|\alpha\|$ and $n \neq 1$. Hence $\lambda_{\omega}$ is bounded. The same argument for $\alpha^{-1}$ proves that $\lambda_{\omega}$ has a bounded inverse.

We will show that $\lambda_{\omega}$ can be taken independent of $\omega$.

LEMMA 3.2. Let $\omega_{1}, \omega_{2} \in M_{*}^{+}$be such that $\operatorname{supp} \omega_{1}$ and supp $\omega_{2}$ are abelian projections. Let $\bar{e}$ be the product (inf) of their central support. Then

$$
\lambda_{\omega_{1}} \bar{e}=\lambda_{\omega_{2}} \bar{e}
$$

Proof. Let $\omega_{i}^{\prime}=\omega_{i} \bar{e}(i=1,2)$. Then $\operatorname{supp} \omega_{i}^{\prime}=\bar{e}$ and

$$
\alpha \omega_{i}^{\prime}=\left(\alpha \omega_{i}\right) \bar{e}=\omega_{i} \lambda \omega_{i} \bar{e}=\omega_{i}^{\prime} \lambda_{\omega_{l}} \bar{e},
$$

where the first equality is by (3.6). Hence $\lambda_{\omega_{l}} \bar{e}=\lambda_{\omega_{l}^{\prime}}$ and we have reduced the problem to the case where the equality

$$
\lambda_{\omega_{1}}=\lambda_{\omega_{2}}
$$

is to be proved for $\omega_{1}$ and $\omega_{2}$ with the same central support.

(1) The case where $\operatorname{supp} \omega_{1}=\operatorname{supp} \omega_{2}$. For $A=\sum_{i=1}^{N} c_{i} e_{i}$ with $N<\infty$, positive numbers $c_{i}$ and central projections $e_{i}$, we obtain by (3.6)

$$
\begin{aligned}
\alpha\left(\omega_{1} A\right) & =\sum c_{i} \alpha\left(\omega_{1} e_{i}\right)=\sum c_{i} \alpha\left(\omega_{1}\right) e_{i}=\alpha\left(\omega_{1}\right) A \\
& =\omega_{1} A \lambda_{\omega_{1}} .
\end{aligned}
$$

Since the $\omega_{1} A$ with such central elements $A$ are norm dense in the set of $\omega \in M_{*}^{+}$with supp $\omega \leq \operatorname{supp} \omega_{1}$ (because supp $\omega_{1}$ is assumed to be an abelian projection of $M$ ), we have $\alpha \omega=\omega \lambda_{\omega_{1}}$, i.e. $\lambda_{\omega}=\lambda_{\omega_{1}}$, for all such $\omega$ and in particular (3.10) holds. 
(2) The case where $\operatorname{supp} \omega_{1} \perp \operatorname{supp} \omega_{2}$. We keep the assumption that $\omega_{1}$ and $\omega_{2}$ have the same central support and supp $\omega_{i}$ are abelian projections. There exists a partial unitary $u \in M$ such that $u^{*} u=$ $\operatorname{supp} \omega_{1}$ and $u u^{*}=\operatorname{supp} \omega_{2}$. Let

$$
\omega_{2}^{\prime}=\omega_{1} \cdot\left(\operatorname{Ad} u^{*}\right) \quad\left(\text { i.e. } \omega_{2}^{\prime}(A)=\omega_{1}\left(u^{*} A u\right)\right) .
$$

Then $\operatorname{supp} \omega_{2}^{\prime}=u u^{*}=\operatorname{supp} \omega_{2}$ and hence $\lambda_{\omega_{2}^{\prime}}=\lambda_{\omega_{2}}$ by (1). Let

$$
\omega(A)=\omega_{1}\left(\left(1+u^{*}\right) A(1+u)\right) / 2 .
$$

For $\lambda_{1}, \lambda_{2}, \lambda \in \bar{e} Z^{+}, Z \equiv M \cap M^{\prime}$, the condition

$$
\omega_{1} \lambda_{1}+\omega_{2}^{\prime} \lambda_{2} \geq \omega \lambda
$$

is equivalent to

$$
\left[\begin{array}{cc}
\lambda_{1} & 0 \\
0 & \lambda_{2}
\end{array}\right] \geq(\lambda / 2)\left[\begin{array}{ll}
1 & 1 \\
1 & 1
\end{array}\right]
$$

i.e.

$$
\lambda \leq\left(\lambda_{1}+\lambda_{2}\right)^{-1}\left(2 \lambda_{1} \lambda_{2}\right)
$$

where inverse is taken on supp $\lambda_{1} \lambda_{2}$. (Because of the support properties, we can restrict our attention to

$$
\left(u^{*} u+u u^{*}\right) M\left(u^{*} u+u u^{*}\right) \approx Z \bar{e} \otimes M_{2}
$$

where $M_{2}$ is $2 \times 2$ matrices.) For given $\lambda_{1}, \lambda_{2}$, there exists the supremum of $\lambda$ in $\bar{e} Z$ given by the right-hand side of (3.16).

Since $\alpha$ is a linear order isomorphism, (3.14) is equivalent to

$$
\alpha\left(\omega_{1} \lambda_{1}\right)+\alpha\left(\omega_{2}^{\prime} \lambda_{2}\right) \geq \alpha(\omega \lambda) .
$$

We note that

$$
\operatorname{supp} \omega=(1+u) e\left(1+u^{*}\right) / 2
$$

is immediately seen to be an abelian projection with the same central support as $e$. By (3.11a), we obtain the equivalence of (3.17) with

$$
\alpha\left(\omega_{1}\right) \lambda_{1}+\alpha\left(\omega_{2}^{\prime}\right) \lambda_{2} \geq \alpha(\omega) \lambda
$$

or equivalently with

$$
\omega_{1} \lambda_{\omega_{1}} \lambda_{1}+\omega_{2}^{\prime} \lambda_{\omega_{2}^{\prime}} \lambda_{2} \geq \omega \lambda_{\omega} \lambda
$$

For given $\lambda_{1}$ and $\lambda_{2}$, the infimum of $\lambda$ satisfying this inequality is

$$
\lambda_{\text {inf }}=\left(\lambda_{\omega_{1}} \lambda_{1}+\lambda_{\omega_{2}^{\prime}} \lambda_{2}\right)^{-1} \lambda_{\omega}^{-1}\left(2 \lambda_{\omega_{1}} \lambda_{1} \lambda_{\omega_{2}^{\prime}} \lambda_{2}\right) \text {. }
$$


(Note that $\lambda_{\omega}^{-1}$ is bounded on $\operatorname{supp} \omega=\bar{e} \geq \operatorname{supp}\left(\lambda_{\omega_{1}} \lambda_{1} \lambda_{\omega_{2}^{\prime}} \lambda_{2}\right)$.) By equivalence of (3.20) and (3.14), this must coincide with

$$
\lambda_{\text {inf }}=\left(\lambda_{1}+\lambda_{2}\right)^{-1}\left(2 \lambda_{1} \lambda_{2}\right) \text {. }
$$

We now obtain

$$
2\left(\lambda_{1}+\lambda_{2}\right) \lambda_{\omega_{1}} \lambda_{1} \lambda_{\omega_{2}^{\prime}} \lambda_{2}=2 \lambda_{1} \lambda_{2} \lambda_{\omega}\left(\lambda_{\omega_{1}} \lambda_{1}+\lambda_{\omega_{2}^{\prime}} \lambda_{2}\right)
$$

for any $\lambda_{1}, \lambda_{2} \in \bar{e} Z^{+}$where $\operatorname{supp} \lambda_{\omega_{1}}=\operatorname{supp} \lambda_{\omega_{2}^{\prime}}=\operatorname{supp} \lambda_{\omega}=\bar{e}$. Therefore

$$
\lambda_{\omega_{1}} \lambda_{\omega_{2}^{\prime}}=\lambda_{\omega} \lambda_{\omega_{1}}=\lambda_{\omega} \lambda_{\omega_{2}^{\prime}}
$$

and hence

$$
\lambda_{\omega_{1}}=\lambda_{\omega_{2}^{\prime}} \quad\left(=\lambda_{\omega}\right)
$$

Since $\lambda_{\omega_{2}^{\prime}}=\lambda_{\omega_{2}}$, we obtain (3.10) for the present case.

(3) The general case. Since $M$ is assumed to be type I without type $\mathrm{I}_{2}$ direct summands in the large, there exists a central projection $E$ such that $M E$ is abelian and $M(1-E)$ is without abelian or type $\mathrm{I}_{2}$ direct summands. As before, we may deal with $M E$ and $M(1-E)$ separately, splitting $\omega \in M_{*}^{+}$as $\omega=\omega E+\omega(1-E)$. For $M E$, the case (1) already proves the lemma.

Now we are left with the case of $M$ without abelian or type $\mathrm{I}_{2}$ direct summands. If $\omega_{1}$ and $\omega_{2}$ are any two elements of $M_{*}^{+}$with a common central support such that $\operatorname{supp} \omega_{1}$ and supp $\omega_{2}$ are both abelian projections, then there exists $\omega \in M_{*}^{+}$with the same central support as $\omega_{1}$ and $\omega_{2}$ such that supp $\omega$ is an abelian projection orthogonal to $\omega_{1}$ and $\omega_{2}$. We can apply the case (2) for the pair $\left(\omega_{1}, \omega\right)$ and for $\left(\omega, \omega_{2}\right)$ to obtain

$$
\lambda_{\omega_{1}}=\lambda_{\omega}=\lambda_{\omega_{2}}
$$

LEMMA 3.3. $\lambda_{\omega}$ can be taken to be independent of $\omega$.

Proof. One can find (by a transfinite induction) a net of $\omega_{\nu} \in M_{*}^{+}$ such that the central support $\bar{e}_{\nu}$ of $\omega_{\nu}$ are mutually orthogonal and their sum is 1 . We define

$$
\lambda_{\alpha}=\sum_{\nu} \lambda_{\omega_{\nu}} \bar{e}_{\nu}
$$

By the argument in the proof of the boundedness of $\lambda_{\omega}$, we have

$$
\|\alpha\| \bar{e}_{\nu} \geq \lambda_{\omega_{\nu}} \bar{e}_{\nu} \geq\left\|\alpha^{-1}\right\|^{-1} \bar{e}_{\nu}
$$


Since $\sum \bar{e}_{\nu}=1, \lambda_{\alpha}$ is bounded with a bounded inverse. It belongs to the center $Z$.

For any $\omega \in M_{*}^{+}$, let $\bar{e}$ be its central support. We have

$$
\lambda_{\omega} \bar{e} \bar{e}_{\nu}=\lambda_{\omega_{\nu}} \bar{e} \bar{e}_{\nu}=\lambda_{\alpha} \bar{e} \bar{e}_{\nu}
$$

for all $\nu$ by Lemma 3.3. Therefore, summing up over $\nu$, we obtain

$$
\lambda_{\omega} \bar{e}=\lambda_{\alpha} \bar{e}, \quad \alpha \omega=\omega \lambda_{\omega}=\omega \bar{e} \lambda_{\omega}=\omega \bar{e} \lambda_{\alpha}=\omega \lambda_{\alpha} .
$$

Combining (3.2) with (3.27) for $\alpha^{\prime}$, we obtain

$$
\alpha \omega=\omega \beta_{\alpha} \lambda_{\alpha}
$$

when supp $\omega$ is an abelian projection. The linear hull of such $\omega$ is norm-dense in $M_{*}$. Since $\alpha$ is linear and norm continuous, we obtain (3.28) for all $\omega \in M_{*}$.

4. Proof of Corollary 1.4. Clearly (1.3) satisfies the required condition for $\alpha$.

To prove the converse, let $\alpha$ be given. Let $\left(M_{i}\right)_{*}^{h}$ be the set of all selfadjoint elements of $\left(M_{i}\right)_{*}$. Each $\omega \in\left(M_{i}\right)_{*}^{h}$ has a unique decomposition

$$
\omega=c_{+} \omega_{+}-c_{-} \omega_{-}
$$

where $\omega_{ \pm} \in\left(M_{i}\right)_{* 1}^{+}$, supp $\omega_{+} \perp \operatorname{supp} \omega_{-}$and $c_{ \pm}$are real positive numbers. We extend $\alpha$ to a bijective map of $\left(M_{1}\right)_{*}^{h}$ onto $\left(M_{2}\right)_{*}^{h}$ by

$$
\alpha \omega=c_{+} \alpha\left(\omega_{+}\right)-c_{-} \alpha\left(\omega_{-}\right) .
$$

We now prove that $\alpha$ is real linear, norm preserving, order preserving and orthogonality preserving.

(1) Linearity. Let $\omega_{1}, \omega_{2} \in\left(M_{1}\right)_{* 1}^{+}$and

$$
\omega=c_{1} \omega_{1}-c_{2} \omega_{2}, \quad c_{1}>0, c_{2}>0 .
$$

With the decomposition (4.1), we have

$$
c_{1} \omega_{1}+c_{-} \omega_{-}=c_{2} \omega_{2}+c_{+} \omega_{+}=c \omega^{\prime}
$$

with $c=c_{1}+c_{-}=c_{2}+c_{+}$and

$$
\omega^{\prime} \equiv\left(c_{1} / c_{+}\right) \omega_{1}+\left(c_{-} / c\right) \omega_{-}=\left(c_{2} / c\right) \omega_{2}+\left(c_{+} / c\right) \omega_{+} \in\left(M_{1}\right)_{* 1}^{+} .
$$

By the affine property of $\alpha$ on $\left(M_{1}\right)_{* 1}^{+}$, we obtain

$$
c_{1} \alpha\left(\omega_{1}\right)+c_{-} \alpha\left(\omega_{-}\right)=c_{2} \alpha\left(\omega_{2}\right)+c_{+} \alpha\left(\omega_{+}\right) \quad\left(=c \alpha\left(\omega^{\prime}\right)\right) .
$$


Hence (4.2) implies

$$
\alpha \omega=c_{1} \alpha\left(\omega_{1}\right)-c_{2} \alpha\left(\omega_{2}\right) .
$$

Combining (4.6) with affine property of $\alpha$, we now obtain real linearity:

$$
\alpha\left(\sum_{i=1}^{N} c_{i} \omega_{i}\right)=\sum_{i=1}^{N} c_{i} \alpha\left(\omega_{i}\right)
$$

for any real $c$ 's and $\omega_{i} \in\left(M_{1}\right)_{* 1}^{+}$and hence for any $\omega_{i} \in\left(M_{1}\right)_{*}^{h}$.

(2) Order. Since $\alpha$ is bijective between $\left(M_{1}\right)_{*}^{+}$and $\left(M_{2}\right)_{*}^{+}$by definition, the linearity implies that $\alpha$ is an order isomorphism.

(3) Orthogonality. This is immediate from the assumption that $\alpha$ on $\left(M_{1}\right)_{* 1}^{+}$preserves the orthogonality.

(4) Norm. From (4.1)

$$
\|\omega\|=c_{+}\left\|\omega_{+}\right\|+c_{-}\left\|\omega_{-}\right\|=c_{+}+c_{-} .
$$

Since $\alpha\left(\omega_{+}\right)$is orthogonal to $\alpha\left(\omega_{-}\right)$(due to the orthogonality of $\omega_{ \pm}$), (4.2) implies

$$
\|\alpha \omega\|=c_{+}\left\|\alpha\left(\omega_{+}\right)\right\|+c_{-}\left\|\alpha\left(\omega_{-}\right)\right\|=c_{+}+c_{-} .
$$

Therefore $\|\alpha \omega\|=\|\omega\|$ for any $\omega \in\left(M_{1}\right)_{*}^{h}$.

By Theorem 1.1, we have

$$
\alpha \omega=\omega \beta_{\alpha} \lambda_{\alpha}
$$

Due to $\|\alpha\|=1,(3.25)$ implies $\lambda_{\omega_{\nu}}=1$ and hence $\lambda_{\alpha}=1$. Therefore there exists a Jordan isomorphism $\beta_{\alpha}$ of $M_{1}$ onto $M_{2}$ satisfying $\alpha \omega=$ $\omega \beta_{\alpha}$ for all $\omega \in\left(M_{1}\right)_{*}^{h}$ and in particular for $\omega \in\left(M_{1}\right)_{* 1}^{+}$.

5. Proof of Proposition 1.6. (1) is already proved in $\S 2$.

Proof of (2) and (3). By a generalized Gleason theorem ([4], [15], [16], [10]) there exists a unique $\alpha_{\gamma} \omega \in M_{*}$ for any $\omega$ satisfying

$$
\left(\alpha_{\gamma} \omega\right)(p)=\omega\left(\gamma^{-1}(p)\right) .
$$

(Here the continuity in $p$ is immediate from the right hand side, cf. [16].) From this definition, $\alpha_{\gamma}$ is linear, maps $M_{*}^{+}$into $M_{*}^{+}$and satisfies

$$
\operatorname{supp} \alpha_{\gamma}(\omega)=\gamma(\operatorname{supp} \omega) \text {. }
$$

Since $\alpha_{\left(\gamma^{-1}\right)}$ is an inverse of $\alpha_{\gamma}$ with the same properties, $\alpha_{\gamma}$ is an o.d. isomorphism. Furthermore, (5.2) implies $\gamma=\gamma_{\alpha}$ for $\alpha=\alpha_{\gamma}$ due 
to (1.4) and due to the uniqueness of the isomorphism $\gamma_{\alpha}$ satisfying (1.4).

Proof of (4). If $\alpha$ is of the form (1.1), let $\gamma$ be defined by $\gamma^{-1}(p)=$ $\beta_{\alpha}(p)$. Then $\gamma$ is an isomorphism of the orthocomplemented lattice $P\left(M_{1}\right)$ onto $P\left(M_{2}\right)$. Since the multiplication of the invertible central element $\lambda_{\alpha}$ does not change the support of the functional $\omega$, we also have

$$
\gamma \operatorname{supp} \omega=\operatorname{supp} \alpha(\omega) \text {, }
$$

namely $\gamma=\gamma_{\alpha}$. Since $\gamma^{-1}(p)=\beta_{\alpha}(p)$, we obtain

$$
\left(\alpha_{\gamma} \omega\right)(p)=\omega\left(\gamma^{-1}(p)\right)=\omega\left(\beta_{\alpha}(p)\right)
$$

by (1.5). This proves (1.6).

Acknowledgment. The author is indebted to the referee for drawing his attention to the reference [7].

\section{REFERENCES}

[1] V. Bargmann, Note on Wigner's theorem on symmetry operations, J. Math. Phys., 5 (1964), 862-868.

[2] A. Barut and A. S. Wightman, Relativistic invariance and quantum mechanics, Nuovo Cimento Suppl., 14 (1959), 81-94.

[3] O. Bratteli and D. W. Robinson, Operator Algebras and Quantum Statistical Mechanics I, Springer-Verlag, Berlin/Heidelberg/New York, 1979.

[4] E. Christensen, Measures on projections and physical states, Comm. Math. Phys., 86 (1982), 529-538. Comments, January 1985.

[5] T. B. Dang and S. Yamamuro, On homomorphisms of an orthogonally decomposable Hilbert space, J. Funct. Anal., 68 (1986), 366-373.

[6] T. B. Dang, On homomorphisms of an orthogonally decomposable Hilbert space, Ph.D. Thesis at ANU.

[7] H. A. Dye, On the geometry of projections in cetain operator algebras, Ann. Math., 61 (1955), 73-89.

[8] R. V. Kadison, Isometries of operator algebras, Ann. of Math., 54 (1951), 325338.

[9] Transformations of states in operator theory and dynamics, Topology, 3 (1965), 177-198.

[10] S. Maeda, Probability measures on projections of a von Neumann algebra (in Japanese), August 1985.

[11] B. Simon, Quantum dynamics: From automorphisms to Hamiltonian, in Studies in Mathematical Physics, ed. E. H. Lieb, B. Simon and A. S. Wightman, Princeton Univ. Press, 1976.

[12] U. Uhlhorn, Representation of symmetry transformations in quantum mechanics, Arkiv Fysik, 23 (1963), 307-340.

[13] E. P. Wigner, Group Theory and its Applications to the Quantum Theory of Atomic Spectra, Academic Press, 1959. (German original 1931). 
[14] S. Yamamuro, On orthogonally decomposable ordered Banach spaces, Bull. Austral. Math. Soc., 30 (1984), 357-380.

[15] F. J. Yeadon, Measures on projections in $W^{*}$-algebras of type $\mathrm{II}_{1}$, Bull. London Math. Soc., 15 (1983), 139-145.

[16] - Finitely additive mesures on projections in finite $W^{*}$-algebras, ibid., 16 (1984), 601-625.

Received February 23, 1988 and in revised form June 16, 1988.

KYOTO UNIVERSITY

KYOTO 606, JAPAN 



\section{PACIFIC JOURNAL OF MATHEMATICS EDITORS}

\author{
V. S. VARADARAJAN \\ (Managing Editor) \\ University of California \\ Los Angeles, CA 90024 \\ Herbert Clemens \\ University of Utah \\ Salt Lake City, UT 84112 \\ THOMAS ENRIGHT \\ University of California, San Diego \\ La Jolla, CA 92093
}

R. FINN

Stanford University

Stanford, CA 94305

HeRmanN FlaschKa

University of Arizona

Tucson, AZ 85721

VAUGHAN F. R. JONES

University of California

Berkeley, CA 94720

STEVEN KeRCKHOFF

Stanford University

Stanford, CA 94305
RoBION KIRBY

University of California

Berkeley, CA 94720

C. C. MOore

University of California

Berkeley, CA 94720

HAROLD STARK

University of California, San La Jolla, CA 92093

\section{ASSOCIATE EDITORS}
R. ARENS
E. F. BECKENBACH
B. H. NeUmanN
F. WolF
K. YosHIDA (1906-1982)

\section{SUPPORTING INSTITUTIONS}
UNIVERSITY OF ARIZONA
UNIVERSITY OF BRITISH COLUMBIA CALIFORNIA INSTITUTE OF TECHNOLOGY UNIVERSITY OF CALIFORNIA MONTANA STATE UNIVERSITY UNIVERSITY OF NEVADA, RENO NEW MEXICO STATE UNIVERSITY OREGON STATE UNIVERSITY
UNIVERSITY OF OREGON UNIVERSITY OF SOUTHERN CALIFORNIA STANFORD UNIVERSITY UNIVERSITY OF HAWAII UNIVERSITY OF TOKYO UNIVERSITY OF UTAH WASHINGTON STATE UNIVERSITY UNIVERSITY OF WASHINGTON 


\section{Pacific Journal of Mathematics}

\section{Vol. 137, No. $1 \quad$ January, 1989}

V. S. Varadarajan, Henry Abel Dye $\ldots \ldots \ldots \ldots \ldots \ldots \ldots \ldots \ldots \ldots$ iii

Huzihiro Araki, An application of Dye's theorem on projection lattices to orthogonally decomposable isomorphisms $\ldots \ldots \ldots \ldots \ldots \ldots \ldots \ldots \ldots$

Richard Arens, The limit of a sequence of squares in an algebra need not be

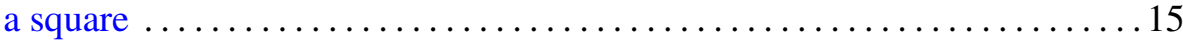

William Arveson, An addition formula for the index of semigroups of

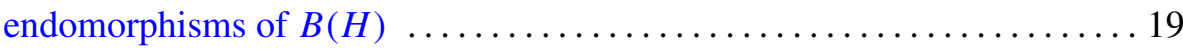

Robert James Blattner and Susan Montgomery, Crossed products and

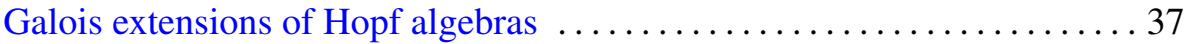

Erik Christensen and Allan M. Sinclair, On the vanishing of $H^{n}\left(\mathscr{A}, \mathscr{A}^{*}\right)$

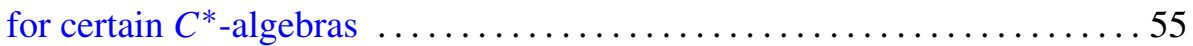

Philip C. Curtis, Jr. and Michael M. Neumann, Nonanalytic functional

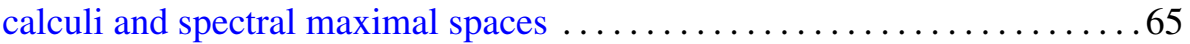

George A. Elliott and David E. Handelman, Addition of $C^{*}$-algebra extensions

Yaakov Friedman and Bernard Russo, Some affine geometric aspects of

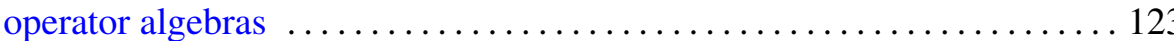

Valentin Ya. Golodets and Sergey D. Sinelshchikov, Regularization of actions of groups and groupoids on measured equivalence relations . . . 145

Irving Kaplansky, CCR-rings 155

Hideki Kosaki, Characterization of crossed product (properly infinite case)

Gert Kjærgaard Pedersen, Three quavers on unitary elements in $C^{*}$-algebras

Sorin Popa, Relative dimension, towers of projections and commuting squares of subfactors

Martin E. Walter, On a new method for defining the norm of

Fourier-Stieltjes algebras 